\title{
Comment on: Assessment of disability in bipolar affective disorder and alcohol use disorder - a descriptive study
}

\author{
J Rohilla
}

\section{Dear Sir,}

This letter bears reference to the study titled “Assessment of disability in bipolar affective disorder and alcohol use disorder - a descriptive study" by S Bondade, CY Sudarshan and S Begum, published in Sri Lanka Journal of Psychiatry, Vol 6 (2) December 2015 (1). All authors deserve appreciation for conducting such a study where disability was assessed in an objective manner in different domain of life in two commonpsychiatric disorders - bipolar affective disorder (BPAD) and alcohol dependence syndrome (ADS). The authors have assessed disability in BPAD not only during episodes of illness but also during the intere-pisodic period.

However, I would like to draw attention of authors to the following issues:

1. The study reports finding a higher degree of disability in the BPAD group as compared to the ADS group. When comparing between these two disorders, it is worth considering that BPAD is an episodic illness but ADS is a not an episodic disorder. So it is not easy to decide whether it is disability during episode or during inter episodic period in BPAD subjects that should be selected to compare with disability in ADS subjects.

2. In the methodology, the authors mention that they excluded subjects with physical and psychiatric conditions. It will be useful to know about nature of physical and psychiatric disorders among the ADS subjects which were excluded from this study. Many psychiatric disorders like delirium tremens, alcohol induced psychosis, alcohol induced cognitive disorder are more common with severe alcohol dependence (2). Similarly, physical disorders like seizures, alcoholic liver disease, alcohol induced gastritis and pancreatitis, neuropathy etc. are more likely in subjects with severe alcohol dependence (2). If ADS subjects with these physical and psychiatric conditions were excluded from study, then it may have affected the quality of the sample of the selected ADS group. Samokhvalov AV, et al., conducted a systematic literature review and expert interviews on the assessment of the relationship between disability and alcohol dependence, and found that psychological and physical symptoms are the major core attributors towards disability related to alcohol use (3). Since a severe degree of alcohol dependence is more likely to result in such psychiatric and physical symptoms, excluding such subjects in the ADS sample might lead to the finding of a lower degree of disability as compared to the BPAD group. Therefore the finding in this study that 'the degree of disability was significantly greater in BPAD patients compared to those with ADS' should be interpreted cautiously, keeping in view the possibility of ADS subjects having severe disability being excluded from the study because of exclusion criterion.

\section{Conflicts of interest}

None declared

J Rohilla, Department of Psychological Medicine, National University Hospital, Singapore

Corresponding author: J Rohilla

Email: jiten.sms@gmail.com

\section{References}

1. Bondade S, Sudarshan CY, Begum S. Assessment of disability in bipolar affective disorder and alcohol use disorder - a descriptive study. SL J Psychiatry 2015; 6(2): 25-29.

2. O’Connell J, Novins DK, Beals J, et al. American Indian Service Utilization, Psychiatric Epidemiology, Risk and Protective Factors Project Team. The relationship between patterns of alcohol use and mental and physical health disorders in two American Indian populations. Addiction 2006; 101(1): 69-83.

3. Samokhvalov AV, Popova S, Room R, Ramonas M, Rehm J. Disability associated with alcohol abuse and dependence. Alcohol Clin Exp Res 2010 Nov; 34(11): 1871-8. 